\title{
Recruiting, Educating, and Taking Primary Care to Rural Communities
}

\author{
Jobn M. Westfall, MD, MPH \\ Hoon Byun, DrPH \\ Robert Graham Center, Washington, DC
}

Ann Fam Med 2020;18:386-387. https://doi.org/10.1370/afm.2601.

$\mathrm{R}$ ural communities face health care inequities not found in urban areas. Most of rural North America is medically underserved. There are just not enough physicians, nurses, physician assistants, and behavioral health clinicians to meet the needs of people living hundreds of miles from the large urban centers that dot our continent. Even in states with higher overall density, some towns and communities still struggle to attract a health care workforce, and where travel to another community for care is fraught with barriers, real and imagined. Rural communities are a heterogeneous group of populations that are both cursed by, and enjoy, the unique interplay of social and cultural differences, economic factors, legislative ignorance, and geographic distance. Apart from the struggle to attract and retain physicians to these communities, the social and health care safety net is also at risk, as public and private organizations find it difficult to reach isolated areas.

How, then, do rural communities solve their health care needs? Of course, the best method for a robust, thriving community is to create and invest in a local community of solution ${ }^{1}$ that can link primary care, behavioral health, public health, and community organizations ${ }^{2}$ to provide the breadth of services from an individual and population health perspective. ${ }^{3}$ What happens while we wait for that? This issue of Annals includes several papers that describe additional methods for delivering care to rural communities.

Conflicts of interest: authors report none.

\section{CORRESPONDING AUTHOR}

John M Westfall, MD, MPH

Robert Graham Center for Policy Studies

in Family Medicine and Primary Care

1133 Connecticut Ave NW

Washington, DC 20036

jwestfall@aafp.org
Waits et al describe the benefits of local obstetrical services on birth outcomes. ${ }^{4}$ They found lower infant mortality in rural Alabama counties with continuous obstetrical services. Obstetrical services are one of the myriad medical services that are becoming less common among family medicine residency graduates. Hospital care, trauma and ER services, and obstetrics are needed in rural communities and this wide array of service may be uniquely provided by a comprehensively trained family physician. In a recent analysis of physicians, it was found that just over 1 in 5 family medicine physicians practiced in 2 or more sites of care, the most common being the clinic and hospital. Increased training may make more comprehensive family physicians available. It is also crucial that rural and remote communities consider ways of focusing their recruitment on comprehensive residency programs and individual residents that have been comprehensively trained. The versatility and ability to move between various sites of care and clinical needs make family physicians good candidates for recruitment into rural communities.

Al Achkar and colleagues describe a virtual psychiatric consult service to support and expand the capacity of local providers to deliver mental health care. ${ }^{5}$ This teleconsultation model provides input into specific patient management, providing invaluable support for local psychiatric care. Through ongoing, systematic case reviews, psychiatric consults become educational opportunities for the local care team. The authors found that this led to greater knowledge of and comfort in providing care for a larger array of mental health conditions. Teleconsult led to expanded access to care.

Pandey et al describe taking the clinic to the community using a mobile free community medical clinic. ${ }^{6}$ The program they describe addresses the cultural barriers often experienced in rural communities. First nation, indigenous communities have faced poor culturally appropriate access to primary care and medical 
services. Free and charitable clinics, which operate just outside the mainstream safety net system, have proven to be especially relevant to rural North Americans. In the United States, despite passage of the Patient Protection and Affordable Care Act, many still find themselves in a coverage gap, as some states have declined Medicaid expansion. Mobile and free clinics help close this gap by providing access to standard primary care and preventive services. Though no 2 clinics are alike, they often take the form of time-limited health fairs and intermittent mobile clinics, and are able to reach populations isolated by geography, language, and culture. Mobile and free clinics face uncertainty, however, as they often rely on time-limited grants and philanthropy. These crucial points of health care access are likely to continue to play a meaningful role in North America and offer opportunities for collaboration with other providers and local organizations to deliver highvalue, patient-centered care to those in need.

These 3 articles remind us of the continuum for addressing rural health care needs. Recruiting and retaining a full-spectrum family physician, along with a team of nurses, physician assistants, and behavioral health clinicians may be the best way to address local health care needs. Add in the occasional social worker, nutrition professional, or peer counselor and it begins to create a team that can address the social determinants of health. Mix in the local public health department, the state extension office, and the smattering of community organizations that care about health, and you have that elusive community of solution. It is not some mythical beast. A community of solution can be built in nearly every community.

On the way to a community of solution, local needs may be addressed by expanding and supporting specialty services through education, consultation, and, occasionally, in-person specialty clinics. Virtual telespecialty services may be enough. They allow specialists to provide care from the relative comfort of their urban office. And, if done correctly, can include synchronous as well as asynchronous care. Specialty consultation done with the care team may be particularly attractive as seen in the study by Al Achkar as it provides individual patient consultation and builds capacity of the local health care team to provide expert mental health services to more people.

When all else fails, sometimes we must take the care to the people, especially in remote areas or in underserved communities of color, indigenous/native communities, and communities with dominant languages other than English, which require a stronger cultural awareness and connection for health care service delivery.

Access to health care in rural communities is fragile, too often dependent on individual clinicians, philanthropy, and lucky recruitment. It may be time for each of us to step up and partner with rural communities in support of their clinical and cultural health care needs, anywhere along the rural health continuum. It is definitely time for the family of family medicine to take seriously the needs of our rural patients and communities.

To read or post commentaries in response to this article, see it online at https://www.AnnFamMed.org/content/18/5/386.

Key words: rural health; primary care; family practice

Submitted July 24, 2020; accepted August 5, 2020.

\section{References}

1. Lesko S, Griswold K, David SP, et al; Folsom Group. Communities of solution: the Folsom Report revisited. Ann Fam Med. 2012;10(3): 250-260.

2 Westfall JM. Cold-Spotting: linking primary care and public health to create communities of solution. J Am Board Fam Med. 2013;26(3): 239-240.

3. Griswold KS, Lesko SE, Westfall JM; Folsom Group. Communities of solution: partnerships for population health. J Am Board Fam Med. 2013;26(3):232-238. 10.3122/jabfm.2013.03.130102.

4. Waits J, Smith L, Hurst D. Effect of access to obstetrical care in rural Alabama on perinatal, neonatal, and infant outcomes: 2003-2017. Ann Fam Med. 2020;18(5):446-451.

5. Al Achkar M, Bennett IM, Chwastiak L, et al. Telepsychiatric consultation as a training and workforce development strategy for rural primary care. Ann Fam Med. 2020;18(5):438-445.

6. Pandey M, Nicolay S, Clark M, et al. Wellness Wheel mobile outreach clinic: a community-led care model improving access to care in indigenous communities. Ann Fam Med. 2020;18(5):466. 